\title{
Child with cerebrospinal fluid rhinorhoea complicated by recurrent meningitis
}

\author{
Samanmali P Sumanasena ${ }^{1}$, Sanath P Lamabadusuriya ${ }^{2}$
}

Sri Lanka Journal of Child Health, 2005; 34: 128-9

(Key words: cerebrospinal rhinorrhoea, recurrent meningitis, child)

\section{Introduction}

Recurrent meningitis in children may be due to immune deficiency, presence of indwelling devices in the ventricular system or breakage of the mucocutaneous barrier between skin and cerebrospinal fluid (CSF). We report a child who presented with recurrent meningitis and CSF rhinorrhoea due to presence of a defect in the cribriform plate.

\section{Case report}

A seven year old boy presented with the seventh episode of pyogenic meningitis in July 2003. He was the second child of non consanguineous parents and was born at term by elective caesarean section with a weight of $2.5 \mathrm{~kg}$. He had multiple dysmorphic features mainly in the craniofacial region (Figure 1) and bilateral congenital sensorineural deafness. The dysmorphic features consisted of bilateral aniridia, microphthalmia and low set ears. There was marked delay in speech but his motor and social functions were age appropriate. Despite aniridia he had useful vision and attended a special school. He had no history of head trauma.

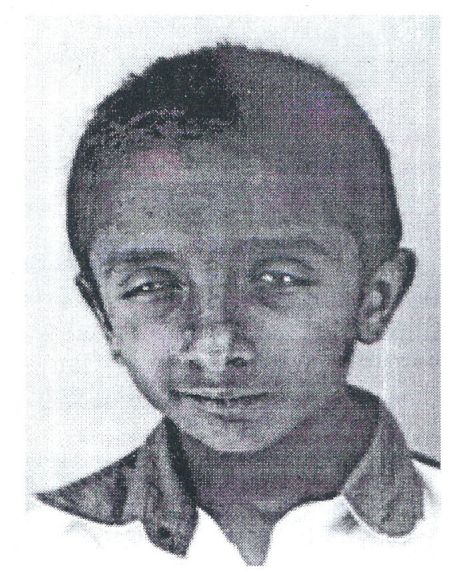

${ }_{1}^{1}$ Lecturer, Department of Paediatrics, ${ }^{2}$ Dean and Senior Professor of Paediatrics, Faculty of Medicine, University of Colombo.

(Received on May 27 2005)
He had initially presented at ten weeks of age with features suggestive of meningitis which were confirmed by CSF analysis. Subsequently almost every year he presented with episodes of pyogenic meningitis which were treated successfully with appropriate antibiotics. Excessive watery discharge from the left nostril had been noted since the neonatal period by his parents. This was confirmed as left sided CSF rhinorrhoea only during the third episode of meningitis at the age of four years when he was extensively investigated for recurrent meningitis. Screening for immune deficiency, including HIV screening, immunoglobulin and complement levels, was normal.

During the first recurrence he was confirmed to have Streptococcal pneumoniae meningitis on CSF culture. Since he was detected to have a CSF leak with microbiologically confirmed streptococcal growth during the fourth episode of meningitis he was started on long term three weekly IM benzathine penicillin prophylaxis with good compliance. He was immunized with 23 valent unconjugated pneumococcal vaccine which was repeated after three years. At the same time he was immunised against meningitis due to Haemophilus influenzae type B and Neisseria meningitidis.

Despite these prophylactic measures he has had a total of nine recurrences. Streptococcal pneumoniae was confirmed on five occasions, including last four episodes, as the causative organism. Since the sixth recurrence these organisms have developed resistance to penicillin resulting in treatment with intravenous vancomicin.

During the fourth recurrence he underwent imaging studies to detect an identifiable anatomical defect with a computed tomography (CT) scan which proved negative. An intrathecal contrast enhanced helical CT scan was done during the sixth recurrence; it consisted of $0.5 \mathrm{~mm}$ coronal sections through the anterior fossa and the paranasal region. Pre and post contrast films were obtained after confirming CSF rhinorrhoea during the procedure. This CT scan was performed following a discussion with a multi 
disciplinary team consisting of a neuro surgeon, an ENT Surgeon and a team of radiologists. Cranial defects in the cribriform plate were confirmed on CT scan by this team of consultants at a joint meeting (Figure 2).

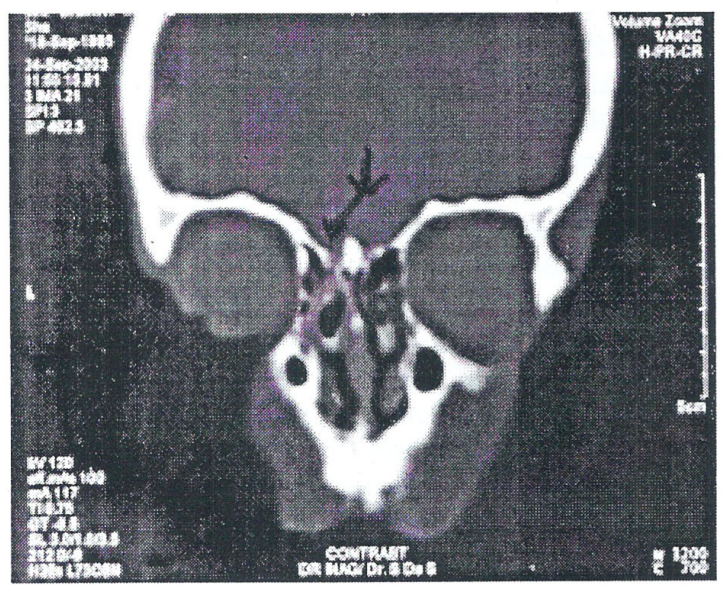

Figure 2. CT scan showing defect in cribriform plate.

The patient underwent an endo-nasal repair of the identified defects in two stages. However he had 2 further episodes of meningitis since then. $\mathrm{He}$ continues to have CSF rhinorrhoea with a remarkable reduction in volume. He was further investigated with a magnetic resonance imaging (MRI) scan to detect residual defects without any success.

\section{Discussion}

Congenital CSF leaks across a mucocutaneous barrier, such as cranial or midline facial defects across the cribriform plate or inner ear fistulae, are associated with increased risk of recurrent pneumococcal meningitis ${ }^{1}$. These leaks are under diagnosed, difficult to locate and carry a potentially lethal cumulative long term risk due to recurrent meningitis. Sensorineural hearing loss and craniofacial anomalies are associated with congenital inner ear anatomical abnormalities. Mondini dysplasia $^{2}$, consisting of peri lymph fistulae connecting the defective inner ear through temporal bone to paranasal sinuses resulting in CSF rhinorrhoea, is one such example. There were no defects detected in the inner ear or temporal bone in our patient.
Nasal endoscopy with intra-thecal fluorescein dye enables location of most of these CSF fistulae and correction with minimal morbidity ${ }^{3}$. An endoscopic technique was used to seal the defect in our patient though intra thecal injection of fluorescein dye was not feasible due to lack of resources. Therefore it is important to maintain possible prophylactic measures to prevent recurrences in this child. Unconjugated pneumococcal vaccine confers capsular specific immunity against 23 sero types in immunologically competent individuals and is the current international recommendation to prevent invasive pneumococcal infection in children more than 5 years of age ${ }^{4}$. Lifelong benzathine penicillin prophylaxis at three weekly intervals to achieve higher drug levels is recommended to prevent invasive infection.

\section{Acknowledgements}

We thank Dr. Sunil Perera, consultant neurosurgeon, Dr. R P Dayawansa, consultant ENT surgeon, Dr. I N A Gunaratne, Dr. S V Alahakoon and Dr. Sanjeewa Munasinghe, consultant radiologists for their invaluable assistance in the management of this patient.

\section{References}

1. Wen HY, Chou ML, Lin KL, Kao PF, Chen JF. Recurrence of pneumococcal meningitis due to primary spontaneous cerebrospinal fluid fistulas. Chan Gung Medical Journal 2001; 11: 724-8.

2. Tullu MS, Khanna SS, Kamat JR, Kirtane MV. Mondini Dysplasia and pyogenic meningitis. Indian Journal of Paediatrics 2004; 71 (7): 6557 .

3. Swift AC, Foy P. Advances in the management of CSF rhinorrhoea. Hospital Medicine 2002; 63: 28-32.

4. Morbidity and mortality weekly report (MMWR) recommendations and reports. Preventing pneumococcal disease among infants and young children. Recommendations of the advisory committee on immunisation practices 2000; 49 (RR-9): 1-35. 